\title{
BMJ Open Public perceptions and experiences of social distancing and social isolation during the COVID-19 pandemic: a UK- based focus group study
}

\author{
Simon N Williams (D) , ${ }^{1}$ Christopher J Armitage, ${ }^{2}$ Tova Tampe, ${ }^{3}$ Kimberly Dienes ${ }^{2}$
}

To cite: Williams SN, Armitage CJ, Tampe T, et al. Public perceptions and experiences of social distancing and social isolation during the COVID-19 pandemic: a UK-based focus group study. BMJ Open 2020;10:e039334. doi:10.1136/ bmjopen-2020-039334

- Prepublication history for this paper is available online. To view these files, please visit the journal online (http://dx.doi org/10.1136/bmjopen-2020039334).

Received 17 April 2020 Revised 06 July 2020 Accepted 07 July 2020
Check for updates

(c) Author(s) (or their employer(s)) 2020. Re-use permitted under CC BY-NC. No commercial re-use. See rights and permissions. Published by BMJ.

${ }^{1}$ Centre for People and Organisation, School of Management, Swansea University, Swansea, UK ${ }^{2}$ Manchester Centre for Health Psychology, University of Manchester, Manchester, UK ${ }^{3}$ World Health Organization, Genève, Switzerland

Correspondence to Dr Simon N Williams; s.n.williams@swansea.ac.uk

\section{ABSTRACT}

Objective This study explored UK public perceptions and experiences of social distancing and social isolation related to the COVID-19 pandemic.

Design This qualitative study comprised five focus groups, carried out online during the early stages of the UK's stay at home order ('lockdown'), and analysed using a thematic approach.

Setting Focus groups took place via online videoconferencing.

Participants Participants $(n=27)$ were all UK residents aged 18 years and older, representing a range of gender, ethnic, age and occupational backgrounds.

Results Qualitative analysis revealed four main themes: (1) loss—participants' loss of (in-person) social interaction, loss of income and loss of structure and routine led to psychological and emotional 'losses' such as loss of motivation, loss of meaning and loss of self-worth; (2) criticisms of government communication-participants reported a lack of trust in government and a lack of clarity in the guidelines around social distancing and isolation; (3) adherence-participants reported high self-adherence to social distancing guidelines but reported seeing or hearing of non-adherence in others; (4) uncertainty around social reintegration and the future-some participants felt they would have lingering concerns over social contact while others were eager to return to high levels of social activity. Most participants, and particularly those in low-paid or precarious employment, reported feeling that the social distancing and isolation associated with COVID-19 policy has had negative impacts on their mental health and wellbeing during the early stages of the UK's 'lockdown'.

Conclusions A rapid response is necessary in terms of public health programming to mitigate the mental health impacts of COVID-19 social distancing and isolation. Social distancing and isolation 'exit strategies' must account for the fact that, although some individuals will voluntarily or habitually continue to socially distance, others will seek high levels of social engagement as soon as possible.

\section{INTRODUCTION}

The current coronavirus (COVID-19) pandemic presents a considerable challenge to public health in the UK and globally. ${ }^{12}$ Pandemics are problematic for clinical and public health agencies and policymakers
Strengths and limitations of this study

- A strength of this this study is that it can help inform social distancing and isolation 'exit strategies', since it provides evidence of how people are likely to behave when these measures are removed or relaxed.

- Another strength of this study is that it is the first qualitative study of its kind to provide evidence on the current mental health impacts of COVID-19related social distancing and isolation.

- Another strength is its finding of the various forms of 'loss' as a new concept through which to understand the practical and emotional impacts of social distancing and isolation on the public.

- A limitation of this study is that it does not include participants from vulnerable or 'high-risk' groups (eg, over 70s)

because of the scientific and medical uncertainty that accompanies novel viruses like COVID-19. ${ }^{3}$ Since COVID-19 is a new virus, pharmaceutical interventions like vaccines are not presently available. Public health policy is therefore exclusively reliant on non-pharmaceutical interventions (NPI). The key NPIs being used in relation to the COVID-19 pandemic in the UK and globally (in addition to personal hygiene advisories; eg, emphasising regular and thorough handwashing) are social distancing and social isolation. Social distancing (physical distancing) in the UK has included the banning of public gatherings, closure of schools and all nonessential shops, workplaces and services, and the recommendation of keeping a distance of $>2 \mathrm{~m}$ apart from others. ${ }^{4}$ Social isolation (self-isolation) guidelines in the UK have included a period of 7 days of quarantine for those showing symptoms of, or testing positive for, COVID-19, and a 14-day quarantine period for others in the same household (although specific guidelines have varied over time). ${ }^{4}$ 
Due to the extent of the social distancing and social isolation measures being implemented in response to COVID-19, social and psychological impacts on the public are anticipated and warrant further attention. ${ }^{5} \mathrm{~A}$ recent rapid review of the psychological impact of quarantine found that longer quarantine duration, infection fears, frustration and boredom, inadequate supplies, inadequate information, financial loss and stigma were among the major stressors. ${ }^{6}$ Another systematic review of the literature on NPIs in relation to pandemic influenza and severe acute respiratory syndrome (SARS) found that people actively evaluate NPIs in terms of criteria such as perceived necessity, efficacy, acceptability and feasibility. ${ }^{7}$ Public views on social distancing and social isolation are ambivalent in some contexts because of their perceived adverse social and economic impacts and their ability to attract stigma, particularly among those required to selfisolate. ${ }^{7}$ Existing research on social distancing and isolation highlights a number of challenges for public health policymakers, including a lack of trust in government ${ }^{8}$; concerns over strains in family resources ${ }^{8}$; gaps and confusions in some areas of pandemic information communication $^{9}$; and low adherence to voluntary social isolation and relatively low adherence to non-attendance at public gatherings. ${ }^{10}$ Although there is existing research from past pandemics on its likely effects, ${ }^{6}$ and new quantitative research is starting to emerge, ${ }^{11}$ there is to our knowledge no published qualitative evidence on public perceptions and experiences of the psychological and social impacts of COVID-19-related social distancing and social isolation, and its relation to adherence. The present study seeks to address this gap.

This study explored four main questions: (1) What are the social and psychological impacts of social distancing and isolation experienced by participants during the COVID-19 pandemic? (2) What are participants' views on government communication around social distancing and isolation? (3) What are participants' current experiences of adherence in relation to social distancing and isolation? (4) What are participants' views on the future in regard to COVID-19 social distancing and isolation? This study therefore aims to contribute to knowledge on adherence to social distancing and isolation policy to provide insight into how communication with the public on social distancing and isolation may be shaped and improved in the future.

\section{PARTICIPANTS AND METHODS}

Five online focus groups with 27 participants were run between 28 March and 4 April 2020. Data were collected 5-12 days after the UK government's 'stay at home' announcement on 23 March 2020, where people were advised to leave their houses only: to shop for basic necessities, take one form of exercise per day, for medical needs, or travelling to work only when necessary and where home working was not possible. Participants were all adults aged 18 years or over currently residing in the

\begin{tabular}{|c|c|}
\hline Characteristic & n (\%) \\
\hline \multicolumn{2}{|l|}{ Gender } \\
\hline Female & $13(48)$ \\
\hline Male & $14(52)$ \\
\hline \multicolumn{2}{|l|}{ Age range } \\
\hline $18-24$ & $7(26)$ \\
\hline $25-34$ & $6(22)$ \\
\hline $35-44$ & $8(30)$ \\
\hline $45+$ & $6(22)$ \\
\hline \multicolumn{2}{|l|}{ Ethnicity } \\
\hline White-British & $16(59)$ \\
\hline White-any other White background & $6(22)$ \\
\hline Asian or Asian British-Pakistani & $3(11)$ \\
\hline Mixed-White and Asian & $1(4)$ \\
\hline Other & $1(4)$ \\
\hline \multicolumn{2}{|l|}{ Occupational classification } \\
\hline Managers, directors and senior officials & $2(7)$ \\
\hline Professional occupations & $6(22)$ \\
\hline $\begin{array}{l}\text { Associate professional and technical } \\
\text { occupations }\end{array}$ & $5(19)$ \\
\hline Administrative and secretarial occupations & $1(4)$ \\
\hline Skilled trade occupations & $1(4)$ \\
\hline Caring, leisure and other service occupations & $1(4)$ \\
\hline Sales and customer service occupations & $3(11)$ \\
\hline Elementary occupations & $1(4)$ \\
\hline Full-time student & $5(19)$ \\
\hline Unclassified/occupation not provided & $2(7)$ \\
\hline
\end{tabular}

Occupational classifications coded using the Office for National Statistics (ONS) Occupation Coding Tool.

UK. Participants were recruited from all four UK countries (England, Wales, Scotland and Northern Ireland). Under normal circumstances, online focus groups can be a useful way of eliciting public views related to matters of health and medicine, particularly from diverse and geographically dispersed participants ${ }^{12}{ }^{13}$ but were necessary due to social isolation policy. Participants gave both verbal and written informed consent.

Purposive sampling was used to provide a diverse range of ages, genders, race/ethnicities and social backgrounds (table 1). ${ }^{14}{ }^{15}$ Researchers used a combination of social media snowball sampling, online community and volunteer advertising sites and social media advertisements (Facebook ads). However, the final sample did consist of a large proportion of white participants and participants aged under 45 years (see the Limitations section for further discussion). Due to social distancing measures, it was necessary for all recruitment to be conducted online.

To ensure that online discussion was manageable, focus groups were kept to between five and eight participants. 
Each group met virtually via a web videoconferencing platform (Zoom) for between 60 and $90 \mathrm{~min}$. Participants joined using both video and audio. All focus groups were organised and moderated by SNW. The topic guide for the focus groups was initially developed using existing literature on public attitudes and experiences in past pandemics and was tested and refined in a pilot focus group. The main topics for the focus groups were: general views on social distancing and isolation; health impacts of social distancing and isolation; views on government COVID-19 advice and communication; and views on compliance with, and the future impacts of, social distancing and isolation.

\section{Analysis}

Data collection and analysis followed an iterative process, whereby emergent themes from early focus groups were used to add to or refine questions during subsequent focus groups. All focus groups were audio recorded and transcribed for coding. SNW and KD analysed the transcripts and developed and applied the thematic coding framework. Themes were discussed and further developed with CA and TT during virtual research group meetings. To help analysis we looked to validate 'sensitive moments' between groups that indicated difficult but important issues. ${ }^{16}$ Negative case analysis was used to seek for information that did not fit emergent themes, and where this occurred, themes were modified accordingly. ${ }^{17}$ Analysis followed a thematic approach as described by Coffey and Atkinson. ${ }^{18}$ Here data analysis takes a pragmatic approach, whereby initial broad research questions inform the abductive generation of themes. ${ }^{18}$ Initial primary (open) codes were developed, and were then developed and connected to other related themes to form overarching secondary codes that were developed into the four themes described below. ${ }^{14} 18$ Data collection and analysis continued until saturation occurred (ie, until no new significant themes emerged).$^{18}$ Data were analysed in NVivo (V.11.4.3, QSR).

\section{Patient and public involvement}

No patients were involved in this study. The public were not involved in the development of the research questions, research design or outcome measures. A pilot focus group with participants not included in the present paper was used to help test and refine the focus group questions. Summary results were disseminated via email to participants prior to publication for feedback and comment.

\section{RESULTS}

Analysis revealed four broad themes: (1) loss; (2) lack of trust in, and clarity of, government communication around social distancing and isolation; (3) high levels of self-adherence but observations of non-adherence in others; and (4) uncertainty around social reintegration and the future. Within each broad theme were a number of substantive subthemes that are discussed below, supplemented by indicative quotes.

\section{Loss}

Many participants felt that the social distancing and isolation polices had had significant social and psychological impacts on their lives, central to which was a feeling of loss. This experience of loss, which one participant likened to a process of 'grieving' (male participant in his 40s), consisted of three practical, social and economic losses: loss of (in-person) social interaction, loss of income and loss of structure and routine. These in turn led to three psychological and emotional 'losses': loss of motivation, loss of meaning and loss of self-worth.

First, participants spoke of a loss of social interaction. The suddenness and extensiveness of the lack of face-to-face contact had, even after only 1 week of lockdown, already 'taken its toll', leaving participants feeling 'alienated':

I've been working at home for the past week and a bit and it's taken its toll ... because you think social contact is such an important part of everyday life and now it's like you walk down the street and people are almost too scared to walk too close. It's so alien. (Male participant in his 20s)

A number talked about feeling depressed or anxious as a result of social distancing or isolation, an experience some likened to 'a prison' (female participant in her 30s) or feeling emotionally 'claustrophobic':

It's all over the news, it's all over your phone, it's all over the TV, it's basically everywhere you turn you are hearing about it. All of a sudden, we can't do these things we used to do, like going to the shops and restaurants, and we just have to stay in, and I think people feel claustrophobic in both a physical and an emotional sense. (Male participant in his 20s)

Second, a number of participants discussed how a loss of income, either through permanent loss of a job, or through temporary loss (via lost clients or customers or being furloughed), had left them feeling 'quite depressed' (female participant in her 30s).

Third, participants expressed a loss of structure and routine, which for some had left them feeling 'less active' or 'sluggish':

I feel really lazy at home. I feel sluggish. I feel out of my routine. I feel much less active, both mentally and physically. You know, not taking the trip to work every day. My working from home schedule is neither here nor there. Mentally I am not as sharp, I feel like I am taking lots of naps in the day. (Male participant in his 20s)

The inability to go to work, or for some the significant restructuring of work patterns, including balancing home working with home schooling, combined with worry over 
the virus itself, meant that many participants felt 'overwhelmed' or 'scared':

I'm literally planning day-to-day as things go along.... I'm not used to having the kids every single day because they are usually at school. It's difficult to work around them, I can't do anything with them, because I can't go out. I feel so scared and don't want to risk it. (Female participant in her 30s)

Participants discussed how impacts like losing their job or not being able to go to work, and not being able to socialise with friends, meant they experienced a general loss of meaning in life:

Being locked in a room trying to find something meaningful to do during the day, and I think it's had a severe impact ... I hope something changes within a few weeks, so I am able to go out and live a fulfilling life. (Male participant in his 30s)

One participant already felt in need of professional mental health support, less than 2 weeks into isolation:

All this talk about social distancing and things is so depressing, terrible, I mean I have even been contemplating on contacting The Samaritans just to be able to try to get through all this. (Male participant in his $30 \mathrm{~s})$

Participants also spoke of a loss of motivation to perform basic everyday tasks, such as personal hygiene and grooming or exercising, and how this demotivation was having an effect on their physical health:

Physically it has had a toll on people. All day you are stuck at home. You eat, you sleep, you work, its gonna have an effect on the body, there is no real drive or motivation. (Male participant in his 20s)

For some, this lack of motivation had left them feeling 'low' or 'depressed':

We are feeling very down and demotivated, very low very depressed to some extent... it's become more stressful to get by and function on a daily basis. (Male participant in his 30s)

Finally, participants suggested that an inability to socialise and the loss of social support led to them feeling a loss of 'self-worth':

Your self-worth goes down a bit, because you can't socialise with people and make yourself feel good about yourself. (Male participant in his 30s)

These emotional and psychological losses were particularly acute for those living in more urban, densely populated cities like London or Birmingham. They were also especially evident among those in low-paid or precarious occupations, who had either lost their job or income or were now relying on parental, familial or state financial support as a result of the pandemic:
The company I work for has closed down and I have had to apply for welfare assistance ... and I've had to go and live with my parents now, and they have had to support me financially. ... it's been difficult, the whole mental health, the ability to function and get by, and being constantly locked in. (Male participant in his 30s)

Lack of trust in, and clarity of, government communication around social distancing and isolation

Most participants felt that guidance on social distancing and isolation had been generally unclear, and that information about the pandemic had conveyed 'mixed messages':

After reading several news publications and channels, there has been much campaign around social distancing, and with isolation you normally associate it when you have got the virus yourself, but I think over the past week there have been several mixed messages over social distancing. (Male participant in his 30s)

Many participants described a lack of trust either in government, who were seen to be 'politicising' the pandemic:

I'm trying to pick my way through what is happening, a lot of politicians are politicising it [COVID-19] and when you read the internet, it is very difficult to know what is real, true or valid, even if you read a broad church of views, facts and figures, it is still very difficult to make sense of it all. (Male participant in his $50 \mathrm{~s}$ )

As noted in the previous section, some participants found that being locked at home coupled with constant media and social media attention on COVID-19 made them feel 'claustrophobic in both a physical and an emotional sense' (male participant in his 20s), and that 'seeing others in a heightened state of anxiety makes it harder to suppress that in yourself' (female participant in her 40s).

Another common criticism was over the ambiguity of terms such as 'essential' and 'emergency' supplies and services:

I have seen loads of people outside, and I wonder how people will enforce that [penalty fines for not social distancing], I'm wondering how can someone prove they are going for an 'emergency reason'? (Male participant in his 30s)

This ambiguity, participants argued, meant that advice was either hard to follow or implement, or that 'loopholes' could be exploited:

Now everyone has been told that they have to stay in their houses, and people are thinking well 'this can be classed as essential, and this can be classed as essential, whereas although we have been told a list of things we can do, people are finding loopholes and 
finding ways to get round them.' (Female participant in her 20s)

\section{High levels of self-adherence but observations of non- adherence in others}

All participants reported being highly adherent to government instructions on social distancing. Participants described how, despite the perceived lack of clarity discussed above, they had been social distancing and isolating as far as possible. Participants also displayed a high degree of social consciousness, with many acknowledging that despite not perceiving themselves as being at high risk, they were doing it to 'save lives' and protect those most vulnerable to the disease:

Staying at home is actually helping to save lives. (Female participant in her 20s)

We have been in lockdown for 14 days, and because of my ... grandmother [in her $80 \mathrm{~s}$ ] who has health problems, it [going out] is just not worth it. (Male participant in his 20s)

Despite reporting their own high degree of adherence, many participants suggested that they had observed instances of non-adherence in others. Observations of non-adherence were associated with three main factors. First, non-adherence was seen to be due to a lack of social conscience. Participants were generally critical of what they perceived to be a minority of 'inconsiderate' individuals:

I'm worried that people are going to take advantage of the nice weather and ruin it for people ... Its insane because they have shut the park, but you get some inconsiderate people like a group of lads playing football or people taking over the paths. (Female participant in her 40s)

This minority of individuals were seen to be flouting guidance related, for example, to public gatherings and not keeping a distance of $>2 \mathrm{~m}$ apart from others when out for daily walks or runs:

The canal path we walk along is not 2 metres wide, but you can just about get around it if you go on the verge and they go on the verge, and most people do but not everybody does ... I don't say anything because ... with all the publicity that's out, if you are still choosing to do that, then me telling you not to do it is not going to make a difference, it's frustrating. (Female participant in her 40s)

Second, non-adherence was seen to be due to a lack of understanding. For example, participants argued that people who were not observing social distancing lacked knowledge over how they could help spread the disease even if they themselves were not exhibiting symptoms:

The vast majority of people are taking it seriously and suffering to a certain extent, but there is a minority who don't necessarily understand it applies to them also. I know of people who have gone to parks or gone for a picnic, because they think 'well we don't know anyone who has any symptoms, and we've not got anything, so we can go about it in the same way.'

(Female participant in her 20s)

Third, non-adherence was seen to be due to a lack of enforcement. Many participants were critical that police were choosing to enforce social distancing restrictions or were not able to (due, for example, to the ambiguity of terms such as 'essential' as discussed in the previous section):

They say that you are not allowed to go out for nonemergency reasons, which I don't think a lot of people are observing. People are just going out whenever they want. Those guidelines are in place ... but noone is really enforcing that. You see police on the street, but they are not really doing anything. (Male participant in his 30s)

Others discussed how, despite their best efforts, supermarkets appeared to struggle to implement social distancing:

The supermarket they are not implementing, what's the point in having the two-metre thing outside when you can't do that inside. ... I went to the supermarket and people respect it outside, but as soon as you go inside there is [sic] people, they don't care, they just come right up to you and try to reach over you. (Female participant in her 40s)

\section{Uncertainty around social reintegration and the future}

According to participants, 'the biggest problem we've got is we don't know when it's going to end' and the sense of 'powerlessness' this had fostered (male participant in his 50s). Despite their high level of current adherence, participants acknowledged there was a limit as to how long they and others could adhere, at least without experiencing more severe social and psychological suffering. Some participants felt that they would rather be told a specific time frame, even if it was far in the future:

I would rather they [the government] said tonight, 'you're gonna be stuck in your houses until September,' than say, 'we will review in three weeks,' and then say, 'we will review in three weeks, and keep doing that,' I'd rather they set a date way in advance in the future because then you can get your head around it. (Male participant in his 20s)

Others feared that while they and others could 'get through' this initial lockdown, having multiple lockdowns (a scenario some knew was possible due to the potential for COVID-19 to re-emerge in a second wave) meant that 'people will really struggle mentally':

I've heard on the grapevine and online sources that we are in this lockdown for a few weeks or so, and then after 12 weeks or so we kind of get released 
and because we are not all immune necessarily it all comes back in a wave and then we have this constant thing of being locked down and then coming out and going back in again, ... and so I think it will go downhill, that's when people will struggle mentally because they've had that taste of freedom, and you don't know how long it's all going to finish. (Female participant in her 20s)

Some felt as though they could not look or plan because anticipating social distancing and isolation over a period of time was 'too overwhelming':

I'm literally thinking day-by-day, because if one was to consider three months of this, and we are only two weeks in, it's just too much, it's too overwhelming. (Male participant in his 50s)

When looking ahead, participants were divided as to how they felt they, and others, would act when social distancing and isolation measures were either relaxed or removed. Some felt that they and others would 'go back to living my life completely as normal' (male participant in his 30 s) as soon as possible. These participants spoke of 'being desperate to go out and go to restaurants or travel a lot' (male participant in his 20s) and generally not taking a graded approach to social reintegration. They argued that if they were 'told its ok' to socially reintegrate, then this was enough for them to "not feel too anxious about going out with friends in the future' (male participant in his 30s). Others felt that it would take them longer to return to prepandemic social behaviours, and, for example, felt that they would continue to have 'anxiety around health' (female participant in her 30s), would be 'cautious' about a 'transition period where I stay in a bit more' (male participant in his 30s) and that people in general might remain 'socially distant' from one another:

People are not going to stay like this for another 6 months. It's for a good reason I know, but it's like a prison, we know what people are suffering mentally and emotionally, we don't know what people are going through behind their door ... When all this comes to an end we don't know how life is going to be. Is everybody going to be socially distant? It's scary.

(Female participant in her 30s)

Others argued that how they would act would likely depend on the circumstances under which social distancing and isolation measures were being relaxed or removed. Specifically, this was tied to their perception of whether COVID-19 still posed a risk to them or to society in general. They argued that, if a vaccine was available, then they would be happy to return to their prepandemic activity.

\section{Alternative accounts and positive perceptions resulting from social distancing and isolation}

Although the findings discussed above represent the most common views exhibited by participants, negative case analysis did reveal some alternative accounts. For example, some participants argued that social distancing and isolation 'hadn't been hard' (male participant in his 20s). However, these participants were all university students, and acknowledged that part of the reason social distancing had not been as difficult for them was there had been no loss of income and, less loss of routine for them (although they had experienced other challenges, for example, related to added university-related stress caused by uncertainty).

A small number of participants argued that they were able to draw positives from the social distancing and isolation due to COVID-19. For example, some described how household quarantine had meant they could have 'more time with their children' (male participant in his 30s) or had brought family units together (male participant in his 50s). However, those participants who explicitly discussed the positives to be drawn from social distancing and isolation were all from higher socioeconomic backgrounds, and tended to live in more rural or less densely populated areas of the UK.

\section{DISCUSSION}

Our findings have explored ways in which many of our participants may be experiencing feelings of anxiety, depression and loss as a result of COVID-19 social distancing and isolation. The social and psychological impacts identified through this study centred around our key theme of 'loss'. Practical social and economic losses-the loss of (in-person) social interaction, loss of income and loss of structure and routine-led to psychological and emotional losses-the loss of motivation, loss of meaning and loss of self-worth. Findings also suggest that participants generally found information on social distancing to be ambiguous. However, there were differing views as to whether the government was at fault (insufficiently clear communication) or that the terms themselves are ambiguous and a small minority of the UK public were taking advantage of the ambiguity. It was recognised that this ambiguity may have been designed to permit greater social freedom than the more extreme social distancing and isolation measures implemented in other countries. Additionally, there was universally high adherence to social distancing and isolation guidelines reported across the study sample, yet most participants had observed or heard of non-adherence in others. Participants were highly critical of such instances of nonadherence, citing lack of social conscience, lack of understanding and lack of enforcement as likely causes. Perhaps the greatest concern for participants was the uncertainty they faced over the duration of the social distancing and isolation measures, as well as their ability to cope longer term. There was also uncertainty as to how they and others would act, with some fearful of lingering inhibitions and anxiety over social contact and health, and others eager to return to normal levels of social activity. 


\section{Relevance to existing literature}

Our findings on COVID-19 social distancing and isolation support some of the findings from existing systematic reviews on previous pandemics related to influenza and SARS. ${ }^{67}$ For instance, we found that frustration or anxiety over loss of social interaction or loss of income, inadequate or ambiguous information, and fears over the duration of social distancing and isolation measures were all major themes. ${ }^{6-8}$ However, contrary to previous research which suggests that adherence with pandemic NPIs is lower in instances where people have low trust in government and where people perceive themselves at relatively low risk from the disease, ${ }^{7}$ our participants were highly adherent to social distancing and isolation measures, despite many lacking trust in government and perceiving themselves at low risk. In fact, stigma was more likely to be attributed to those who were failing to socially distance and isolate. Of course, as noted above the scale and severity of the pandemic and subsequent measures are unprecedented. As such, although there is existing research on its likely effects, ${ }^{6}$ and although quantitative research is starting to emerge, ${ }^{11}$ there is, to our knowledge, no published qualitative evidence on public perceptions and experiences of the psychological and social public experiences of COVID-19-related social distancing and isolation, and its relation to adherence-a gap this study addresses.

\section{Limitations}

One limitation of this study is that it is not possible to rule out that the high degree of adherence and social conscience that participants expressed was not at least partly affected by social desirability bias, which can often be encountered in focus group studies. ${ }^{19}$ However, conducting focus groups online has been found to reduce social desirability bias (although it is worth noting that this is more so where asynchronous or text-only communication is used, and not videoconferencing as in our study). ${ }^{12} 20$

Another limitation is that the sample was not as diverse as was desired, in terms of ethnicity, with a number of ethnicities (including those from Black or Black British ethnicities) not represented. This was due to the fact that no participants from these ethnic groups applied to take part in the study. Future research will seek to recruit a more ethnically diverse sample. Also, this study did not recruit participants who are deemed at particularly high risk from COVID-19-related complications, for example, individuals aged 70 and over and those living with certain chronic health conditions (no participants were aged over 60 years old).$^{21}$ Because these individuals are likely to have been significantly affected by social distancing and isolation policy (being required to self-isolate for 12 weeks), their views will be important. It is also worth noting that our recruitment material did encourage those at high risk to apply, though we received no applications from those over 70 . This may be partly due to the fact that those over 70 are a hard-to-reach group online, because they are significantly less likely to use social media or be heavy internet users, ${ }^{22}$ which, due to the lack of online social support and interaction, might mean they are at particularly high risk of some of the negative social and psychological impacts discussed in this paper. Future research will explore at-risk groups' experiences in depth. Future papers will also explore further the similarities and differences in views and experiences in the perceptions of experiences of participants living in different parts of the UK (eg, London compared with less densely populated areas), a theme only briefly discussed here due to limitations of scope.

\section{Implications for policy and practice}

This study suggests that for some, the social distancing and isolation associated with COVID-19 policy may have had negative impacts on mental health and well-being. The prevalence of COVID-19-related depression and anxiety, and the extent to which it will last beyond the removal or relaxation of social distancing and isolation policies remains to be seen. Our ongoing research will explore these social and psychological impacts longitudinally. Policymakers and the public health community must discuss measures to respond to the likely wave of mental ill health which is expected to follow, and which is tentatively suggested by our early qualitative evidence. The theme of loss and addressing public concerns around physical and emotional losses (eg, meaning and self-worth) may inform current and future therapeutic interventions. Loss of meaning and self-worth may be due in part to loss of control, and increasing a sense of control for the public should be considered in future policy, intervention and programming. ${ }^{23}$ Additionally, findings suggest that a rapid response is necessary in terms of public health programming to mitigate these mental health impacts. Waiting until restrictions and isolation measures are relaxed or removed to provide support services could potentially have devastating impacts. Government and the public health authorities should look at ways of extending mental health outreach services, especially remotely. ${ }^{24}$ Timely attention is needed for those who are predisposed to depression and anxiety, those who may be suicidal and those experiencing significant social, economic and personal loss.

Our study also suggests that the COVID-19 pandemic has disproportionately negatively affected those in lowpaid or precarious employment. Future research and policy should therefore seek to develop measures that specifically seek to remediate the social, economic and psychological harms related to COVID-19 as experienced by those from disadvantaged backgrounds. Looking ahead to later stages in the current pandemic, or to the development of pandemic preparedness programmes for the future, a couple of lessons can be distilled, which warrant urgent attention. First, initial high levels of support for, and adherence to, social distancing and isolation measures are likely to wane over time, particularly where end dates are and remain uncertain. Second, in planning the 'exit strategy' for the UK lockdown, and its 
possible impact on future resurgences of COVID-19 infection, policymakers and public health authorities need to account for the fact that, although some individuals will voluntarily or habitually continue to socially distance (graded social reintegration) others will seek immediately to reintegrate fully beyond what they are permitted to.

Contributors All authors (SNW, CA, TT, KD) contributed to the planning of the study. The analysis was conducted by SNW and KD. The initial draft of the article was written by SNW. All authors (SNW, CA, TT, KD) revised the manuscript and approved the final version for publication. SNW is the guarantor of the article.

Funding This research was supported by the Manchester Centre for Health Psychology based at the University of Manchester (£2000) and a 'Greatest Need Fund' award (£3000) from Swansea University. CA's contribution was additionally supported by the NIHR Manchester Biomedical Research Centre and the NIHR Greater Manchester Patient Safety Translational Research Centre.

Disclaimer The funders played no role in the conduct of the study.

Patient and public involvement Patients and/or the public were not involved in the design, or conduct, or reporting, or dissemination plans of this research.

Patient consent for publication Not required.

Ethics approval Ethical approval was received by Swansea University's School of Management Research Ethics Committee (SOM-REC-STAFF-091).

Provenance and peer review Not commissioned; externally peer reviewed.

Data availability statement Data are available upon reasonable request. Ethical restrictions related to participant confidentiality prohibit the authors from making the data set publicly available. During the consent process, participants were explicitly guaranteed that the data would only be seen my members of the study team. For any discussions about the data set please contact the corresponding author: SNW (s.n.williams@swansea.ac.uk).

Open access This is an open access article distributed in accordance with the Creative Commons Attribution Non Commercial (CC BY-NC 4.0) license, which permits others to distribute, remix, adapt, build upon this work non-commercially, and license their derivative works on different terms, provided the original work is properly cited, appropriate credit is given, any changes made indicated, and the use is non-commercial. See: http://creativecommons.org/licenses/by-nc/4.0/.

ORCID iD

Simon N Williams http://orcid.org/0000-0003-2854-9946

\section{REFERENCES}

1 Prime Ministers' Office. PM statement on coronavirus. Available: https://www.gov.uk/government/speeches/pm-statement-oncoronavirus-12-march-2020

2 Ferguson N, Laydon D, Nedjati-Gilani G. Impact of nonpharmaceutical interventions (NPIs) to reduce COVID-19 mortality and healthcare demand, 2020. https://www.imperial.ac.uk/media/ imperial-college/medicine/sph/ide/gidafellowships/Imperial-CollegeCOVID19-NPI-modelling-16-03-2020.pdf

3 Williams SN. 'Plausible uncertainty': The negotiated indeterminacy of pandemic influenza in the UK. Crit Public Health 2008;18:77-85.

4 National Health Service (NHS) UK. Guidance on social distancing. Available: https://www.gov.uk/government/publications/covid- 19-guidance-on-social-distancing-and-for-vulnerable-people/ guidance-on-social-distancing-for-everyone-in-the-uk-andprotecting-older-people-and-vulnerable-adults [Accessed $20 \mathrm{Mar}$ 2020].

5 Holmes EA, O'Connor RC, Perry VH, et al. Multidisciplinary research priorities for the COVID-19 pandemic: a call for action for mental health science. Lancet Psychiatry 2020;7:547-60.

6 Brooks SK, Webster RK, Smith LE, et al. The psychological impact of quarantine and how to reduce it: rapid review of the evidence. The Lancet 2020;395:912-20.

7 Teasdale E, Santer M, Geraghty AWA, et al. Public perceptions of non-pharmaceutical interventions for reducing transmission of respiratory infection: systematic review and synthesis of qualitative studies. BMC Public Health 2014;14:589.

8 Baum NM, Jacobson PD, Goold SD. "Listen to the people": public deliberation about social distancing measures in a pandemic. Am J Bioeth 2009:9:4-14.

9 Akan H, Gurol Y, Izbirak G, et al. Knowledge and attitudes of university students toward pandemic influenza: a cross-sectional study from turkey. BMC Public Health 2010;10:ar412.

10 Mitchell T, Dee DL, Phares CR, et al. Non-pharmaceutical interventions during an outbreak of 2009 pandemic influenza A (H1N1) virus infection at a large public University, April-May 2009. Clin Infect Dis 2011;52 Suppl 1:S138-45.

11 Geldsetzer P. Knowledge and perceptions of COVID-19 among the general public in the United States and the United Kingdom: a crosssectional online survey. Ann Intern Med 2020:M20-0912.

12 Tates K, Zwaanswijk M, Otten R, et al. Online focus groups as a tool to collect data in hard-to-include populations: examples from paediatric oncology. BMC Med Res Methodol 2009;9:ar15.

13 Williams SN. A twenty-first century Citizens' POLIS: introducing a democratic experiment in electronic citizen participation in science and technology decision-making. Public Underst Sci 2010;19:528-44.

14 Mays N, Pope C. Rigour and qualitative research. BMJ 1995;311:109-12.

15 Kitzinger J. Qualitative research. introducing focus groups. BMJ 1995;311:299-302.

16 Barbour R, Kitzinger J. Developing focus group research: Politics, theory and practice. London: Sage, 1999.

17 Silverman D. Qualitative research: theory, method and practice. London: Sage, 1997.

18 Coffey A, Atkinson P. Making Sense of Qualitative Data. London: Sage, 1996.

19 Albrecht T, Johnson G, Walther J. Understanding communication processes in focus groups, in D Morgan (ED.) successful focus groups: advancing the state of the art. London: Sage, 1993.

20 Stewart K, Williams M. Researching online populations: the use of online focus groups for social research. Qualitative Research 2005;5:395-416.

21 National Health Service (NHS) UK. Advice for everyone: coronavirus (COVID-19). Available: https://www.nhs.uk/conditions/coronaviruscovid-19/ [Accessed 24 Mar 2020].

22 van Boekel LC, Peek ST, Luijkx KG. Diversity in older adults' use of the Internet: identifying subgroups through latent class analysis. $J$ Med Internet Res 2017;19:e180.

23 Ryan R, Patrick H, Deci E, et al. Facilitating health behaviour change and its maintenance: interventions based on Self-Determination theory. The Eur Health Psychol 2008;10:2-6.

24 Rushton K, Fraser C, Gellatly J, et al. A case of misalignment: the perspectives of local and national decision-makers on the implementation of psychological treatment by telephone in the improving access to psychological therapies service. BMC Health Serv Res 2019;19:997. 